\title{
Comparative study of inter-and intrahemispheric cortico-cortical connections in gerbil auditory cortex
}

\author{
HARDY THOMAS ${ }^{1}$ and VLADIMIR LÓPEZ ${ }^{2}$
}

${ }^{1}$ Instituto de Fisiología, Facultad de Medicina, Universidad Austral de Chile, Valdivia, Chile.
${ }^{2}$ Escuela de Psicología, Pontificia Universidad Católica de Chile, Santiago, Chile.

\begin{abstract}
Topographic distributions and laminar pattern of cortico-cortical projections from the primary auditory field (AI), anterior auditory field (AAF), dorsoposterior field (DP), ventroposterior field (VP), dorsal field (D) and ventral field (V) were studied in relation to tonotopic maps in combined anatomical, electrophysiological and 2-deoxyfluoro-D-glucose (2DG) experiments. Distributions of axons were examined by means of retrogradely-transported fluorescent tracer Fast Blue (FB) injected in the primary $(\mathrm{AI})$ and anterior $(\mathrm{AAF})$ auditory field. Injections of fluorescent tracer were placed in electrophysiologically-identified locations of AI and AAF. Neurons in AAF, DP, VP and V project to AI in the ipsilateral hemisphere. This area also receives projections from AI, AAF and D from the contralateral hemisphere. In AI, DP and VP, neurons are connected with AAF in the ipsilateral hemisphere and AI and AAF in the opposite hemisphere. In all cases, patches of labeling are distributed along 2DG bands oriented parallel to the isofrequency line. Substantial numbers of retrogradedly labeled neurons with similar best frequencies (BFs) were observed in the ipsilateral and moderate to scant numbers in the contralateral hemisphere. In general, regions near the injection sites receive more densely-labeled projections than do more distant targets. In both hemispheres, the supragranular layer III contains the greatest concentration of cortico-cortical cells bodies; the granular and infragranular layer V contains a somewhat lower concentration.
\end{abstract}

Key terms: auditory cortex, cortical connections, laminar patterns, fluorescent tracer-retrograde labeling.

\section{INTRODUCTION}

Anatomical and physiological studies have greatly expanded our knowledge of the organization of the mammalian auditory cortex. Previous electrophysiological mapping studies have provided evidence that the rodent auditory cortex, like that of other mammals, consists of multiple topographically-organized cochlea projections. The auditory cortex of the Mongolian gerbil has been studied extensively using electophysiological recording techniques (Thomas et al., 1993), 2-deoxyglucose (2DG) autoradiographic labeling (Scheich et al., 1993) and anterograde tracer biocytin (Budinger et al., 2000). Six fields were distinguished on the basis of frequency representation. Four different tonotopically- organized fields could be distinguished: two fields with a mirror-imaged tonotopy had adjacent high frequency representations, the low frequency representation were rostral in a smaller anterior field (AAF) and caudal in a large field (AI). More caudally, additional fields were found, a dorsal field (DP) and a ventral posterior field (VP). A dorsal field (D) was identified dorsocaudal to AI. A tonopic organization of field D could not be discerned, neither could a ventral field (V) located ventral to the common highfrequency border of AAF and AI. For a detailed schematic representation see Figure 1.

From a comparative point of view, this multiplicity of cortical auditory areas is a feature present in most mammalian species, independent of their auditory capacities 


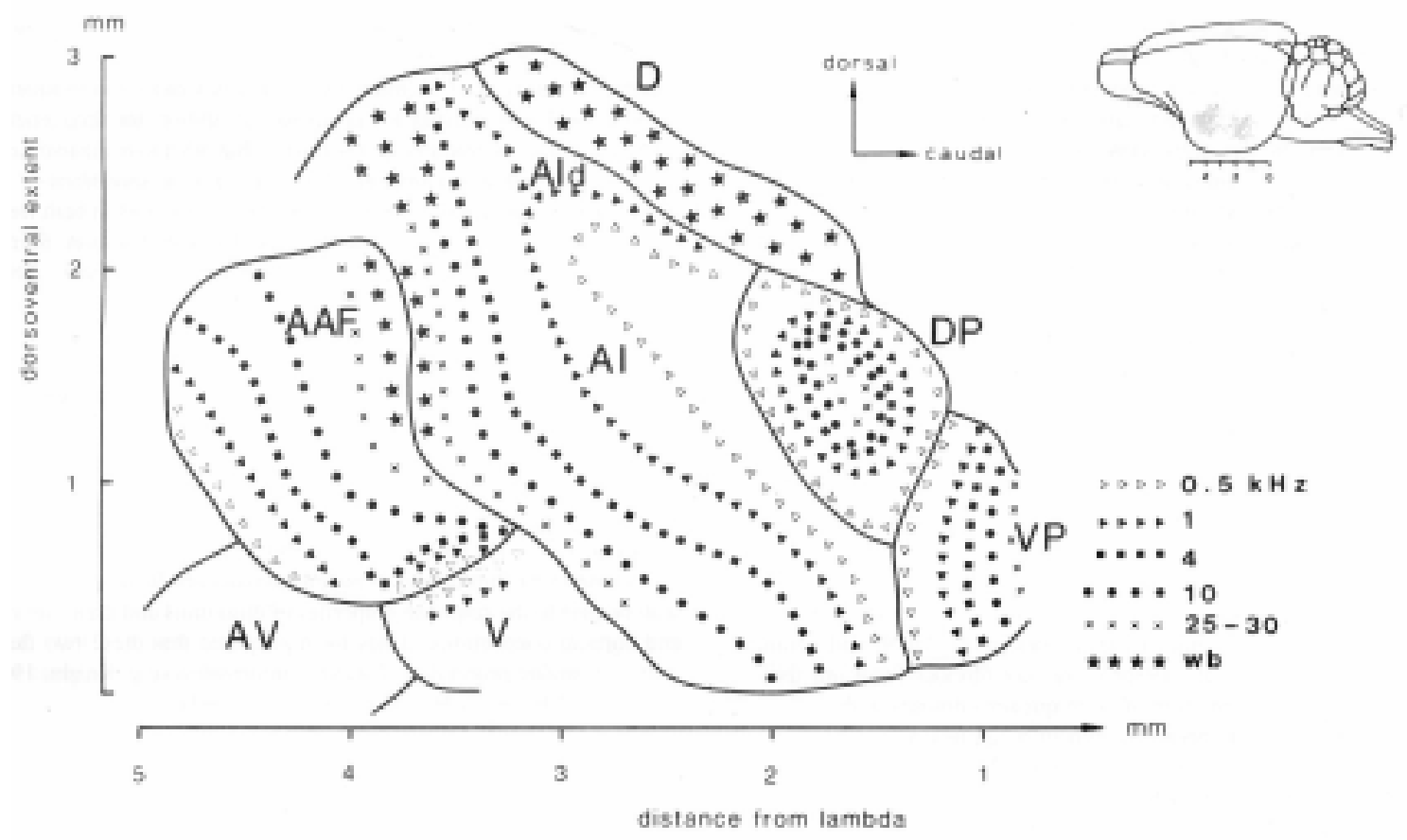

Figure 1

Schematic summary of the frequency organization of the gerbil's auditory cortex in the left hemisphere (From Thomas et al., 1993).

(Brugge and Reale, 1985; Ehret, 1997; Rouiller, 1997; Rauschecker, 1998).

The present paper provides a morphofunctional analysis of the auditory cortex of the Mongolian gerbil using electrophysiological, 2-deoxyglucose and pathway retrograde tracing techniques in parallel to describe the projections of AI and AAF upon the auditory fields in the ipsilateral and contralateral hemispheres. In addition, we describe features of topographic and laminar distribution of axons seen in individual cortical target fields after AI and AAF injection.

\section{METHODS}

The results described in this study were obtained from 17 adult, male and female, gerbils (Meriones unguiculatus) weighing 60-80 g. The animals were initially anaesthetized with urethane, $1.4-1.8 \mathrm{~g} / \mathrm{kg}$ i.p., followed by supplementary doses given as required to maintain light anesthesia during the recording. After removing the skin and temporal muscle, the upper part of the temporal cortex was exposed, leaving the dura intact, and covered with an oily erythromycin-cortisone mixture. Before extracellular Fast Blue (FB) injection, a short electrophysiological mapping had to be performed to identify the Best Frequency (BF) and to confirm in which auditory field (AAF or $\mathrm{AI}$ ) the injection site was to be located. The details of the FB technique, used in this work, can be found in Bentivoglio et al. (1980).

Multiunit recordings were made with a glass-insulated Elgiloy-electrode. Tone bursts used for stimulation had durations of 250 or $500 \mathrm{~ms}$. Free-field sound was presented to the ear contralateral to the recording side by a speaker and a high frequency sper, placed about $10 \mathrm{~cm}$ from the pinna. A nearly constant sound pressure level (SPL) of $65 \pm 5 \mathrm{~dB}(60-70 \mathrm{~dB}$ in the frequency range $0.1-35.0 \mathrm{kHz}$ ), was calibrated by a Bruel \& Kjaer sound level meter at the position of the animal head. Selection of frequency was in a pseudorandom fashion, with 5 to 10 computer- 
generated repetitions. Spike events were displayed as dots on a line. The dotted displays and their integration in the form of isointensity-frequency histograms were recorded and saved. BFs of units were determined from the peak 'on' responses in histograms. BFs maps of the cortex were obtained from each brain and used to guide the placement of tracer injections. In order to obtain discrete zones of FB application, the tracer was deposited in its crystalline form by means of glass micropipette tips (outside diameter $40 \mathrm{um}$ ) filled with crystalline FB. Later, the electrophysiological mapping was also used as reference to corroborate the 2deoxyglucose (2DG) autoradiographic labeling.

After a survival time of 7 days following the microelectrode mapping and fluorescent tracer injection, the gerbil was injected i.p. with 18-20 uCi of 2-deoxyfluoro-D-glucose $(14 \mathrm{C}(\mathrm{U}))$ (Amersham-Buchler) in $0.2 \mathrm{ml}$ sterile saline and placed in a small cage with a carpet floor to reduce sound production from movement. Stimulus exposure lasted 90 minutes in a lighted soundproofed chamber. Stimuli were delivered free-field from loudspeaker above the cage. Stimulus intensity was $75 \pm 5 \mathrm{~dB}$ SPL for all frequencies used, as measured at the floor of the cage. The gerbils were stimulated with tones of two different frequencies that were generated under computer control by a Rockland synthesizer. One frequency was similar to the BF injection sites.

After the 90-minute exposure, the animals were decapitated, and the brains were removed and frozen in a cryostat. In each series of sections, every third horizontal slice was processed in the following manner:

i) Fluorescent microscopy: cells labeled with fluorescent tracer were plotted under ultraviolet light with a Leitz-Ortoplan microscope coupled to an X-Y plotter;

ii) Serial $40 \mu \mathrm{m}$ frozen sections were dried at $50^{\circ} \mathrm{C}$ onto slide and placed on contact slides with Kodak NMB X-ray film for two weeks. The prints were carefully cut out following the cortical surface, and the two hemispheres were divided. Each hemisphere was reconstructed by mounting consecutive sections on millimeter graph paper using the rostral tip of the hippocampus as a zero reference for rostro-caudal alignment of sections;

iii) The cytoarchitectural organizations of the auditory cortex were studied by using conventional Nissl stains with cresyl violet

\section{RESULTS}

The possibility that connections between the different auditory fields exist can be demonstrated by comparing the results obtained after the injection of FB (retrograde tracer) with the results from 2deoxyglucose autoradiographic labeling.

Here, we present the data from the injections of FB made in the primary (AI) and anterior (AAF) auditory fields of 17 gerbils. Table I lists these animals, along with information about the injection sites and the resulting transport observed for each case. Seventeen injections performed in 17 gerbil were placed in tonotopically organized fields: twelve in AI $(\mathrm{BFs}=0.6$ $\mathrm{kHz}, 0.8 \mathrm{kHz}, 1.0 \mathrm{kHz}, 1.2 \mathrm{kHz}, 1.4 \mathrm{kHz}$, $1.6 \mathrm{kHz}, 3.2 \mathrm{kHz}, 3.6 \mathrm{kHz}, 5.0 \mathrm{kHz}$ and 15 $\mathrm{kHz})$ and five in AAF (BFs $=1.0 \mathrm{kHz}, 1.5$ $\mathrm{kHz}, 2.5 \mathrm{kHz}, 3.0 \mathrm{kHz}$ and $5.6 \mathrm{kHz}$ ). These BFs and injections sites were confirmed in each field by $2 \mathrm{DG}$ isofrequency contours.

\section{Cortico-cortical connection}

The results revealed a complex pattern of intrinsic connections in AI and AAF fields, ipsilateral connections with at least five regions of the auditory cortex and callosal connections with homotopic and heterotopic auditory fields of the contralateral cerebral hemisphere. Figures 2-5 summarize the major features of these ipsi- and contralateral projections patterns and indicate the tonotopic organization of the temporal surface of the hemisphere as previously determined electrophysiologically (Thomas et al., 1993) and by 2DG mapping (Scheich et al., 1993). 


\section{TABLE I}

Summary of the extent of Fast blue labeling in ipsilateral and contralateral auditory fields following injection into AI and AAF.

Primary Auditory Field (AI)

\begin{tabular}{llclc}
\hline $\begin{array}{l}\text { Reference } \\
\text { number }\end{array}$ & $\begin{array}{c}\text { Injection site } \\
\text { layers }\end{array}$ & $\begin{array}{r}\text { Best frequency } \\
(\mathrm{kHz})\end{array}$ & \multicolumn{1}{c}{$\begin{array}{c}\text { Summary of Fast blue transport } \\
\text { ipsilateral }\end{array}$} & $\begin{array}{c}\text { AI-D } \\
\text { contralateral }\end{array}$ \\
\hline G166 & I-II-III-IV & 1.6 & AAF-D (DP-VP) & AI-AAF-D \\
G167 & I-II-III-IV-V-VI & 0.8 & AAF-D (DP-VP) & AI \\
G96 & I-II-III & 0.6 & AAF-D (DP-VP) & AI \\
G100 & IV-V & 0.8 & AAF & AI-D \\
G101 & VI & 3.2 & D-V & AI-D \\
G102 & II-III-IV & 15.0 & AAF (D) & AI-AAF \\
G103 & III-IV & 5.0 & AAF-D-V & AI-D \\
G104 & III-IV & 1.0 & AAF-VP & AI \\
G112 & II-III & 3.6 & AAF-DP (D) & AI (D) \\
G128 & IV-V & 1.2 & AAF & \\
G129 & III-IV-V & 15.0 & AAF-DP-VP (D) & AAF-D \\
G164 & I-II-III & 1.4 & & AAF-AI \\
Anterior Auditory Field & & & AAF-AI \\
G105 & I-II-III-IV-V & 1.0 & AI-DP-VP & AAF \\
G144 & I-II-III & 1.5 & AI-DP-VP & AAF-AI \\
G148 & I-II-III-IV & 5.6 & AI-DP-VP & AAF \\
G149 & I-II-III-IV-V-VI & 3.0 & AI-DP-VP & AI-DP-VP \\
G165 & III-IV-V & 2.5 & &
\end{tabular}



Figure 2

Reconstruction of cortical sections after Fast Blue tracer injection in the central region in AI by 0.6 $\mathrm{kHz}$ in layers I-II-III. The ipsilateral projections show labeled cells in AAF, D and in the ventral region between AI and AAF (ventral field). From the caudal fields retrogradely-labeled cells were found mainly in the ventral part of this region. In the contralateral hemisphere, labeled cells were found only in the homotopic field AI. In this experiment, the gerbil was stimulated with two tones of $1.0 \mathrm{kHz}$ and $8.0 \mathrm{kHz}$. 


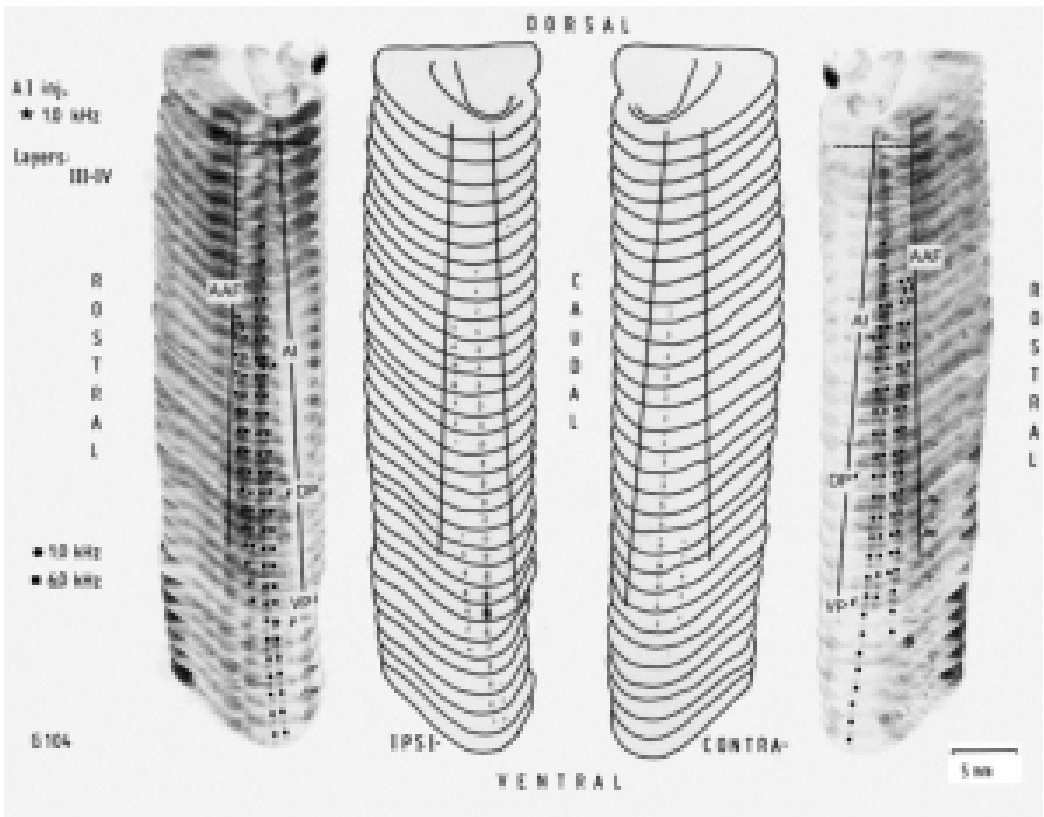

\section{Figure 3}

Tracer injection was placed in the ventral region in AI by $1.0 \mathrm{kHz}$ in layers III-IV. The ipsilateral projections from AAF show two separate patches. Labeled cells were found in contralateral homotopic field and the ventral region in AAF.

\section{Figure 4}

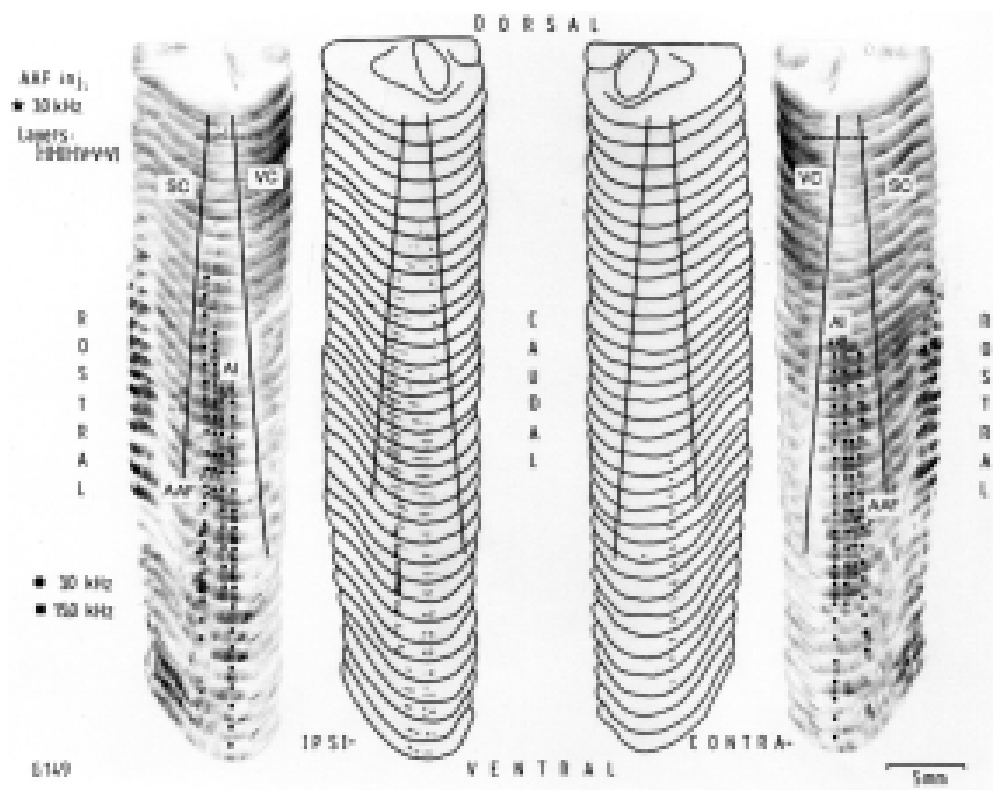

Tracer injection was placed in the central region in AAF by $3.0 \mathrm{kHz}$ in layers I, II, III, IV, V and VI. After the injection in AAF, the intrinsic projections showed two retrograde patterns: one ran parallel to the isofrequency contours and the second was seen as a large elongated patch of labeling oriented in a rostrocaudal direction, orthogonal to the isofrequency contours. The ipsilateral projections from AI also showed a labeled band orthogonal to the direction of the low-to-high best frequency gradient. Labeled cells are seen in the ventrocaudal and dorsal region of AI. In the contralateral hemisphere, homotopic labeling was found along with a small number of labeled cells in the heterotopic field of AI. 


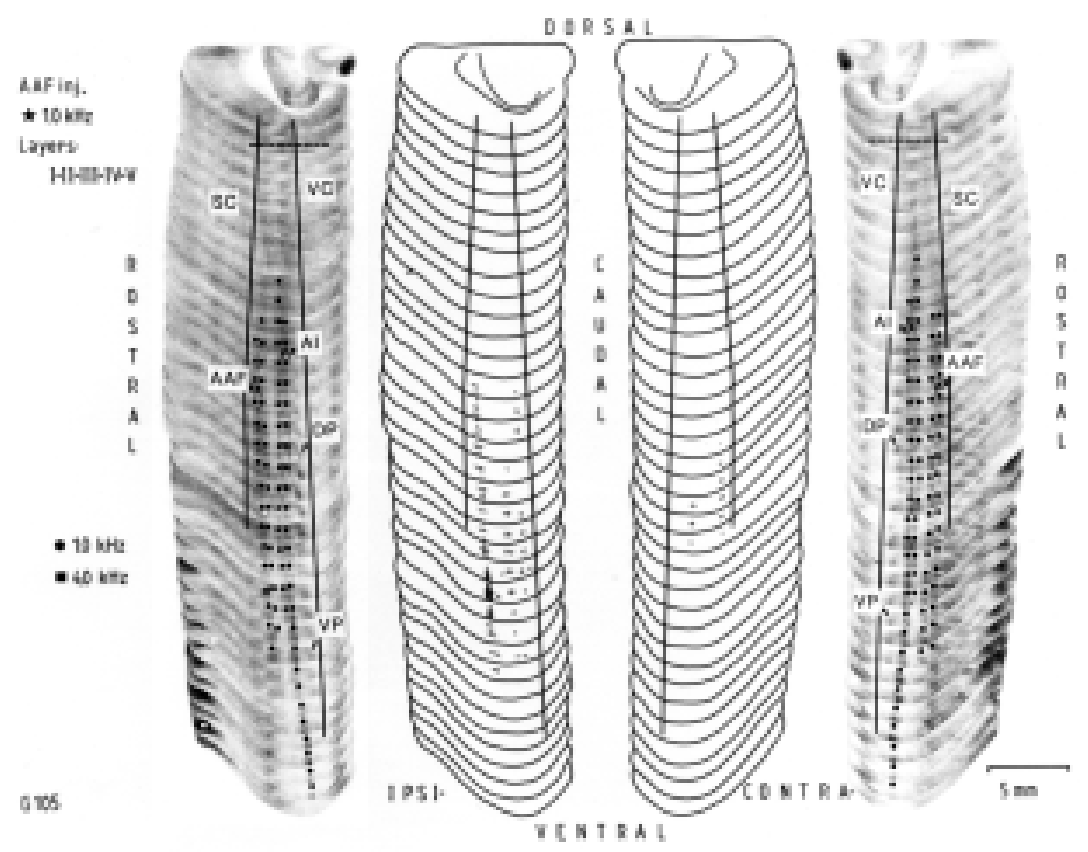

\section{Figure 5}

Injection in the central region of AAF by $1.0 \mathrm{kHz}$, in the layers I, II, III, IV, V and VI. Projections from AI and from caudal fields DP and VP are visible. The opposite hemisphere show labeled cells in the homotopic field and in the heterotopic field AI.

\section{Figure 2 to 5}

Reconstruction of the left and right hemispheres in the region of the auditory cortex from horizontal autoradiographs and parallel serial reconstruction of horizontal sections containing labeled cell bodies (each black dot indicates the position of one labeled cell) in the auditory cortex. Sections were aligned along a dorsoventral reference with the hippocampus. The strongest band of labeling in the auditory cortex with a dorsoventral orientation in the autoradiography corresponds to the isofrequency contours of 2DG and are indicated by different symbols. The contralateral hemisphere corresponds to the right hemisphere and the left hemisphere to the ipsilateral where the injection tracer was placed (indicated by a black star in the autoradiographs' horizontal hemisection).

SC: somatosensory cortex VC: visual cortex

\section{Distribution of the intrinsic projection in AI} and $A A F$

We also studied the distributions across the cortical surface in sections of tissue cut horizontally and tangential to cortical layers in $\mathrm{AI}$ and AAF. In AAF, intracortical patches were usually found within $1 \mathrm{~mm}$ from the center of the injection site, and the interpatch spacing varied between 300 and $400 \mathrm{um}$. In all such cases, the patches were found in regions with $2 \mathrm{DG}$ isofrequency contours or equivalent to the BFs of the cells within the injection site. Variations observed in the labeling patterns correlated with the individual variability in the direction of elongation of the isofrequency contours across the cortical surface. Figures 2-5 illustrate typical patterns of isofrequency contours found in AI and AAF. Isofrequency contours in AAF ran roughly parallel to those in AI, oblique to dorsoventral cortical axis, with some irregularities seen in the individual contours themselves.

In $\mathrm{AI}$, the maximal distance of labeled clusters from the injection center can be identified in experiments in which the injection focus is confined to layers III-IV. Here, we found a vertical elongated chain of $\mathrm{FB}$ that was in the range of $2.5 \mathrm{~mm}$; the 
labeled cell bodies were found through layers III to $\mathrm{V}$, gerbil $96(0.6 \mathrm{kHz})$, gerbil $104(1.0 \mathrm{kHz})$ (Figs 2 and 3). In AAF, when the injection spans all six layer of the cortex, the intrinsic projections were generally found in layers II-III and in the upper part of layer V. After these relatively large injections in AAF, two retrograde patterns were observed: one ran parallel to isofrequency contours; and the second was a large elongated patch of labeling found oriented in a rostrocaudal position, orthogonal to isofrequency contours, gerbil $149(3.0 \mathrm{kHz})$, gerbil 105 $(1.0 \mathrm{kHz})$ (Figs 4 and 5).

\section{Injections into the primary auditory field (AI)}

The primary auditory field is located in a central position within the auditory cortex and has the greatest extent among all auditory fields. Labeling patterns after FB injection into AI are shown in Figures 2 and 3.

\section{Projections from the anterior auditory field $(A A F)$}

In most experiments, it was established that AI had connections with the anterior auditory field. The only exception was in gerbil $101(3.3 \mathrm{kHz})$ which did not show retrograde transport into $\mathrm{AAF}$. In this animal, the injection site was in the superior portion of layer V. In gerbil 104 $(1.0 \mathrm{kHz})$, gerbil $164(1.4 \mathrm{kHz})$ and gerbil $96(0.6 \mathrm{kHz})$, two separated patches are evident, showing ipsilateral projections from AAF to AI. Two dense columns of labeling can be seen within ipsilateral AAF (Figs 2 and 3) separated by a more lightly labeled area. This pattern was also present in gerbil $167(0.8 \mathrm{kHz})$.

These patterns of FB labeling appeared regularly following injections into layers I to IV, but in tracer injections confined to infragranular layers (V-VI), a diffuse labeling pattern is apparent.

Distributions of labeled cells showed exact correlations with tonotopic organizations in AAF. In those cases in which the injection site was in a high $\mathrm{BF}$
$(15.0 \mathrm{kHz})$, the labeled cells were located in the dorsocaudal region in AAF (gerbil 102 and gerbil 129). On the other hand, injection sites with low BFs $(0.6 \mathrm{kHz})$ showed retrograde labeled cells bodies in the dorsoventral portion of AAF (Fig 2). Within the anterior auditory field, layers III and $\mathrm{V}$ showed labeled cells, but staining was more intense in layer III independently of the injection site in the AI cortex.

\section{Projection from the dorsoposterior (DP) and ventroposterior (VP) fields}

In the caudal region of the auditory cortex, a retrograde pattern was observed in several experiments: gerbil $167(0.8 \mathrm{kHz})$, gerbil $166(1.6 \mathrm{kHz})$, gerbil $104(1.0 \mathrm{kHz}$, Fig 3) and gerbil $129(15.0 \mathrm{kHz})$. Differences in the projection patterns were shown when the injection sites in AI were different from the $\mathrm{BF}$ representations.

Following injections in low BFs, gerbil $96(0.6 \mathrm{kHz}$, Fig 2), gerbil $167(0.8 \mathrm{kHz})$ and gerbil $166(1.6 \mathrm{kHz})$, only one patch of transported labeling was found on the caudal region to AI. After the FB tracer was injected into the high best-frequency (15.0 $\mathrm{kHz}$ ) representation of AI (gerbil 129 and gerbil 102) an axonal transport was not found in this region. In gerbil 104 (1.0 $\mathrm{kHz}$ ), the injection tracer was in the ventral portion of AI; here, one label was observed in the ventroposterior auditory field (Fig 3). In the caudal field of the cortex, more labeled cells were found in layer III.

\section{Projections from the dorsal (D) and ventral (V) fields}

Although some experiments showed labeled cells in both the dorsal and ventral region of AI, the majority showed retrograde transport in the dorsal region of AI. In gerbil $102(15.0 \mathrm{kHz})$, the injection was placed in AI's dorsal portion, and it was unclear whether the labeling patterns should be assigned to isofrequency contours of the dorsal region of AI or to the dorsal field. In gerbil 103, gerbil 101, gerbil 96 (Fig 2) and gerbil 164, the ventral region between AI 
and AAF revealed connection patterns with AI. In both fields, labeling was more heavily concentrated in layers III and IV.

\section{Callosal connections of AI}

After injections of tracer into AI, labeled neurons were found in several locations of the contralateral hemisphere. For most injections, dense foci of labeled cells were found in contralateral homotopic field in AI and also in the heterotopic dorsal field. Rostral injections tended to label more rostral locations in the contralateral cortex while caudal injections labeled more caudal contralateral sites. In the gerbil AI, low Best Frequencies are represented caudally and high Best Frequencies rostroventrally. The rostroventral portion of AAF projects to the caudal portion of AI and the caudal portion of AAF projects to the rostral portion of AI, in the contralateral hemisphere. These results suggest that portions with similar frequency representation in $\mathrm{AAF}$ and $\mathrm{AI}$ are connected between hemispheres. Projections to AI from the other contralateral fields (DP, VP, V) were not found. In the opposite hemisphere, cell bodies in AAF, AI and D are concentrated in layers III and $\mathrm{V}$, similar to the projection seen from AAF to $\mathrm{AI}$ in the ipsilateral hemisphere.

\section{Injections into the anterior auditory field $(A A F)$}

The anterior auditory field (AAF) is located in the rostral part of the auditory cortex and is the second largest auditory representation. Both, AAF and AI were clearly distinguished by their reversed gradients of best frequencies. In AAF high BFs were found caudally, near the highfrequency representation of AI, and low BFs rostroventrally. Following injections into AAF, we detected labeled axons in $A I, D P$ and $V P$ in the ipsilateral hemisphere and in AI and AAF in the contralateral hemisphere. Labeling patterns in AAF after $\mathrm{FB}$ injections are shown in Figures 4 and 5.

\section{Projections from AI}

Heavy labeling was seen in AI following FB tracer injections into ipsilateral anterior auditory field. Often, some labeled regions in AI appear elongated in a direction that parallels the low-to-high gradient of the best-frequency map. On the other hand, comparisons of the $2 \mathrm{DG}$ patterns with the best-frequency maps obtained in the same experiment led to the conclusion that the FB labeled bands are oriented in the direction of the isofrequency contours. Although elongated patches of label were present in all AI fields of each hemisphere we examined, individual patterns of labeling varied. One feature of the pattern that differed between the hemispheres was the orientation of the elongated patches with respect the isofrequency contours. In gerbil $144(1.5 \mathrm{kHz})$ and gerbil 149 (3.0 $\mathrm{kHz}$ ) (Fig 5), an elongated band of labeling is oriented parallel to the low-to-high gradient of the best frequency, and another narrow band runs parallel to the isofrequency contour in the ipsilateral hemisphere. In the opposite hemisphere, only light to moderate labeling that runs in a dorsoventral direction was found (Figs 4 and 5). In all experiments, following FB injections in AAF, we found projections from $\mathrm{AI}$ in which $\mathrm{FB}$ are more heavily distributed in the upper layer $\mathrm{V}$.

\section{Projections from the caudal fields (DP and $V P$ )}

The heaviest distributions of labeled cells were found in the region caudal to AI, gerbil $149(3.0 \mathrm{kHz})$ (Fig 4). In gerbil 149 $(3.0 \mathrm{kHz})$ and gerbil $105(1.0 \mathrm{kHz})$ (Figs 4 and 5), two long, separated bands of labeling crossing the caudal fields were identified. Comparison of the labeling patterns with the best-frequency maps obtained in the same experiments led to the conclusion that the labeled bands were oriented parallel to the direction of the lowto-high gradient of best frequencies in DP and VP caudal fields. An overlapping distribution of labeled cells was visible in layer III (See Fig 6). 


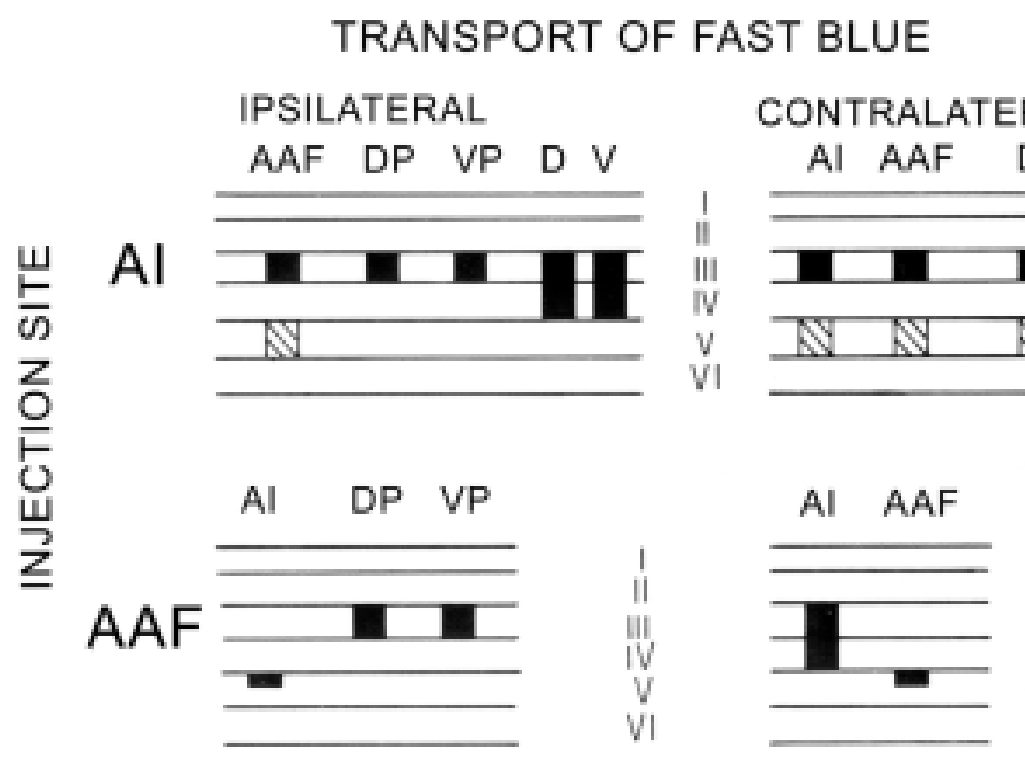

\section{Figure 6}

Summary diagram of the cortical projections from AI and AAF. Projections from fields AI and AAF are shown upper and lower respectively. Projections from the ipsilateral fields are depicted in the column on the left, and projections from the contralateral fields are shown in the column on the right. Densely-labeled cortical laminae are shown as solid black; moderately-labeled laminae contain diagonal lines.

Projections from the dorsal (D) and ventral (V) fields

Compared with injections into AI, we found few retrogradedly-labeled neurons following injections into AAF. In gerbil $149(3.0 \mathrm{kHz})$ (Fig 4), the neurons were located in the ventral region between AAF and AI. Projections from the dorsal field were not labeled. However, in gerbil $165(2.5 \mathrm{kHz})$ we detected diffusely-distributed labeled cells in the dorsal region to AI.

\section{Callosal connections to AAF}

After injections of tracer into AAF, we found homotopic labeling in the corresponding contralateral field and heterotopic labeling in AI. Rostral injections tended to label more rostral locations and caudal injections more caudal sites in the contralateral cortex (Figs 4 and $5)$. The most dense concentration of labeled cells in the opposite hemisphere was among the pyramidal cells of layer III and the granular layer IV.

A relative smaller number of labeled cells was found in the heterotopic field in AI, gerbil $144(1.5)$, gerbil $149(3.0 \mathrm{kHz})$ and gerbil $105(1.0 \mathrm{kHz})$. In these cases, labeled cells were more heavily distributed in upper layer III.

\section{DISCUSSION}

The present work reveals an exact correlation between the cortico-cortical connections in different auditory fields and the so-called BFs of the auditory cortex in rodents. The cortical organization of projections is described with reference to a detailed sequence of horizontal cuts. The main results of this work show that ipsi and contralateral projections in the gerbil auditory cortex exhibit a pattern as complex as those described for more evolved mammals like cats and monkeys (Imig and Reale, 1980; Fitzpatrick and Imig, 1980; 
Matsubara and Phillips, 1988; Cipolloni and Pandya, 1989; Morel et al., 1993).

The mosaic-like projections include those that are intrinsic in $\mathrm{AI}$ and $\mathrm{AAF}$, as well as others between different auditory fields and the BFs that showed an almost invariant organization in the series of experimental animals. Nevertheless, tracer injections into different cortical layers or in a different number of layers resulted in different densities of labeled cells or projections. Parallel to electrophysiology and tracer injection, the tonotopic organization was assessed using a 2DG method to assure the exact position and distribution of trace labeling. This allowed an exploration of the correlations between anatomy, electrophysiology and the $2 \mathrm{DG}$ contours.

In the 2DG reconstruction, the isofrequency contours distribution for $\mathrm{AI}$ and $\mathrm{AAF}$ were clearly identified, as were those of the caudal fields (DP and VP). The primary auditory field $\mathrm{AI}$ and the anterior auditory field (AAF) of the gerbil have a mirrored organization of their BFs maps as has been shown previously electrophysiologically (Thomas et al., 1993) and by means of 2DG autoradiographies (Scheich et al., 1993). In both fields, the isofrequency contours run parallel in a dorsoventral direction and and, ventrally, turn in a more caudal direction. Using the radioactive labeling by $2 \mathrm{DG}$, it was possible to accurately localize the injection site, the auditory field and the BFs in the gerbil auditory cortex.

\section{Intrinsic Connections}

The neuroanatomic experiments reported here confirm the model of neocortex organization and its connection patterns. By intrinsic horizontal connections we mean all those projections that run parallel to the cortical surface within the layers of an auditory cortical field. These connections can project in different directions, in the case of the gerbil, either dorsoventrally or rostrocaudally. In addition to the horizontal connections, vertical projections are distributed in different layers and run perpendicular to the cortical surface.

Numerous cellular properties are developed within and between cortical areas thanks to these spatially-segregated intrinsic connections. They form true excitatory and inhibitory neuronal networks that allow multiple neural interactions. These interactions represent a central topic in most neuroanatomic research programs where the main goal is to identify the intrinsic interactions in a vertical sense, i.e., vertical interlayer interactions. Nevertheless, the present work shows that horizontal interlayer connections also may play a very important function in stimuli processing and integration at a cortical level.

In AI, the frequency organization is exhibited as thin bands or strips that run parallel to the isofrequency contour. Injection into infragranular layers showed the shortest extension of horizontal connections with a maximal distribution of $1.4 \mathrm{~mm}$. Injections in the granular and supragranular layers of AI resulted in projections of greater extension (2.2 $\mathrm{mm}$ approximately).

Combined experiments of electrophysiology and radioactive tracer (proline and leucine) in cats showed active foci isolated from the injection site (Imig and Reale, 1980). Similar findings in the same animal were later reported using retrograde microinjections with WGA-HRP in AI (Matsubara and Phillips, 1988). These author found isolated labeled areas as far as $3 \mathrm{~mm}$ from the injection site. Such projections were observed both in pyramidal and non-pyramidal cells from the III and IV cortical layers.

These spot-shaped labeled areas in the cat cortex are clearly different from the rather continuous pattern within the isofrequency contour observed in the gerbil. Nevertheless in some of our experiments with gerbils, especially when tangential cuts were used, a sort of discontinuity in the isofrequency contours could be seen on the 2DG studies.

In layers II, III and V of the gerbil AAF, a pattern of projections with a dorsoventral direction coexisting with a distribution of projections in a rostrocaudal direction was observed, orthogonal with the low-high gradient of frequencies in this area. Morphological research of the auditory cortical cells in the cat, using the Golgi 
technique, showed that axons and dendrites are both oriented preferentially in the axis of isofrequencies (Winer, 1984b). Additionally, neurons in layer III showed large horizontal branches (Winer, 1984a; 1984b). Results of the present work suggest that mainly pyramidal cells participate in the horizontal interaction processes. However, a concrete cellular study could only be done using the Golgi impregnation method, which was beyond the scope of the present research.

The horizontal intrinsic connections might represent an structural characteristic feature in all mammals species. It is not surprising that such functional mechanisms could play a relevant role in sensory information processing, not only in the neocortex but in the paleocortex as well.

The labeling pattern in the isofrequency contours observed with the 2DG method and with electrophysiologic recordings suggest that, in the gerbil AAF, a gradient of BFs exist which is oriented in rostrocaudal direction. The width of the area with labeled cells is greater than the contours of a single BF. This could lead us to hypothesize that there is a functional organization with two patterns of connections: one large, horizontal pattern oriented in the same direction as the isofrequencies, and another, more physiological or functional connection with different directions into the same field that might have an effect on extrinsic connections with other areas.

\section{Corticocortical Connections}

In all the studied, animals anatomical and physiological methods were combined to assess the relationship between BFs and the connections in the gerbil auditory cortex. Three main questions about the gerbil auditory cortex extrinsic connections were intended to be answered in the present work:

The topography of extrinsic projections in $\mathrm{AI}$ and $\mathrm{AAF}$;

The labeling pattern in those fields connected with AI and AAF; and

The distribution of labeled cells in the layers of the auditory cortex fields.

\section{Connections to AI}

Descriptions of the pattern of ipsi- and contralateral connections in the temporal cortex of different species have shown that all neurons in the identified auditory fields are connected with cells from the primary auditory fields (Fitzpatrick and Imig, 1980; Imig and Reale 1980; Aitkin et al., 1988; Luethke et al., 1989; Morel et al., 1993). The present results in gerbils show that AI is interconnected ipsilaterally with AAF, $\mathrm{DP}, \mathrm{VP}, \mathrm{D}$ and $\mathrm{V}$ and has retrograde contralateral connections with $\mathrm{AI}$ and AAF. Additionally, this pattern of projection is organized in such a way that regions representing the same frequencies are connected both ipsi- and contralaterally.

\section{Projections from $A A F$}

An auditory field anterior to AI has been described in different species using anatomic and electrophysiologic methods. This is the $\mathrm{S}$ field in the guinea pig (Redies et al., 1989; Wallace and Rutkowski, 2002), the R field in the squirrel (Luethke et al., 1988), the A field in the cat (Imig and Reale, 1980), the R field in the owl monkey (Imig et al., 1977) and the RL field in the Macaque monkey (Merzenich and Brugge, 1973). In the gerbil, after FB injection in AI two to three foci of labeled cells were found in the so-called $\mathrm{AAF}$, within the isofrequencies contours labeled with 2DG. Intermixed regions less densely labeled were observed between these sites. Spot-shaped labeling patterns previously have been described in the somatosensory cortex (Jones et al., 1978), in the visual cortex (Montero, 1980), and in the auditory cortex (Brugge and Imig, 1978) of the monkey and the cat. These patterns were discussed as a general characteristic of cortico-cortical connections. In cats, another significant and discontinuous labeling was observed in an anterior field (A) after radioactive tracer injection in AI (Imig and Reale, 1980). These multiple spots were observed in the cortex as columns running from the cortical surface to the white matter. In the gerbil, there are labeled cells in AAF that project to AI especially to layers III and 
$\mathrm{V}$ with more intense labeling in layer III. The tonotopic organization of $\mathrm{AAF}$ in the gerbil (low frequencies distributed rostrally and high frequencies caudally) as well as the inverse frequency gradient in AI is clearly reflected in the projection pattern of AAF. Thus, the rostral region of AI connects with the caudal region in AAF and caudal region of AI with the rostral AAF. In the gerbil, as well as in cats, frequency-specific connections have been found between AI and AAF showing the same tonotopic organization (Imig and Reale, 1980). In the owl monkey, an inverse tonotopic map is evident with a common limit in the low frequencies between AI and the rostral field; projections from AI caudal region to $\mathrm{R}$ caudal region are identified in this animal. In conclusion, both evidences from the gerbil as well as from other species support direct interactions between cells sharing the same BFs through topographically-organized corticocortical connections.

\section{Caudal fields projections}

Cortico-cortical connections between AI and the caudal fields were observed in several of our experiments, but this occurred only when injections were located in the low-frequency regions. The labeled cells were principally located in layer III. Tracer injection in high- and mediumfrequency regions of AI did not show transport to the caudal fields. The cause for this is unknown yet, but the scarce representation of high frequencies in the caudal fields might play an important role. In other words, the labeling of the few neurons representing medium and high frequencies in the caudal field might not be easily seen.

Electrophysiologic recordings of this region showed two well-organized tonotopic fields, but the projections from these fields to AI run only in the dorsoventral direction. This could be explained by the fact that the low-frequency region is common for DP, VP, and the primary auditory field. Something similar was described in cats where the low frequencies are located in the limit between AI, P and
VP (Reale and Imig, 1980). In this animal, when radioactive tracer was injected in the low-frequency regions of $\mathrm{AI}$, a continuous unique label was seen from the $\mathrm{P}$ field to VP (Imig and Reale, 1980).

\section{Projections from $V$ and $D$ fields}

We found connections between $\mathrm{AI}$ and $\mathrm{V}$ fields only in three experiments. The $\mathrm{V}$ field seems to be very similar to the cat's AII field located ventrally in the auditory cortex.

Ipsi- and contralateral labeling were frequently observed in the D field (dorsal to AI). When injections were placed in AI low-frequency regions or close to the limit between $\mathrm{AI}$ and $\mathrm{D}$, it was impossible to separate the labeled areas due to the continuous low-frequency gradient between these fields. However, in these same experiments two different labeled areas were easily identified in the contralateral hemisphere. These could be reflecting a smaller number of projections from the contralateral D field compared to ipsilateral neurons.

\section{Contralateral projections to AI}

Callosal connections between AI and its homonymous contralateral field as well as with the heterotopic D contralateral field were observed as a rule in every experiment, while contralateral connections to AAF were not always easily identified. The contralateral labeling in AI occurred more densely in layer III but also in layer $\mathrm{V}$. These results match others obtained after WGA-HRP injections in gerbil auditory cortex where pyramidal cells from layers III and $\mathrm{V}$ were described as the origin of transcallosal projections (Budinger et al., 2000). Previous works report that callosal neurons in several species are located in layer III. For example, lesion experiments in cats showed anterograde contralateral degeneration in layer IV and the lower part of layer III (Diamond et al., 1968). Using HRP, retrograde-labeled cells were observed in layers III and IV (Imig and Brugge, 1978). In the owl monkey, auditory 
callosal projections were found in the upper part of layer III and in layer IV (Fitzpatrick and Imig, 1980). Using HRP, callosal connections were early described in rodents in layers III and V (Jacobson and Trojanowski, 1974). In the somatosensory cortex of the rat, most callosal neurons were localized in layers III and V. The contralateral projections from AAF in gerbil clearly show a similar orientation between the pattern of labeled cells and the isofrequency contours defined by $2 \mathrm{DG}$. Similar findings have been reported in cats following the injection of radioactive material in the AI field of the contralateral hemisphere (Imig and Reale, 1980).

In the gerbil, cells that project through from AAF, AI and D to the contralateral AI are located in layers III and V, with more intense labeling in layer III. Another important characteristic of these projections is that those which are homotopic have a denser labeling than the heterotopic ones. This findings was also described for the cat (Imig and Reale, 1980).

\section{Connections with AAF}

Fast Blue injections in AAF showed ipsilateral connections with AI and caudal fields DP and VP. After Fast Blue injection in AAF, the pattern of labeled areas in AI was comprised of bands running in the same direction of the isofrequency contour in some experiments. However, in other experiments labeled bands also ran orthogonally to the isofrequency lines or parallel to the low-tohigh frequency gradient.

In the cat, ipsilateral projections from $\mathrm{A}$ and $\mathrm{P}$ to $\mathrm{AI}$ run along the low-frequency gradients where a high density of axonal ends can be found. Projections from the anterior A field match exactly with columns whose cells exhibit dominant contralateral suppression (Imig et al., 1982). In addition, studies conducted in owl monkeys showed two types of labeling patterns in AI: a lighter labeling involving layers I, II, V and VI and another, denser labeling in layers III and IV (Fitzpatrick and Imig, 1980). In the gerbil, after the injection in AAF this label tag was especially evident in the upper part of layer $\mathrm{V}$.

\section{Caudal field projections}

In some cases, projections in the caudal fields were more numerous than those seen in the AI projection studies. Nevertheless, this pattern was difficult to identify. Previous works with other species report that close areas or regions exhibited denser labeling than more distant areas or fields (Imig and Reale, 1980). In the present work. the labeled areas observed after AAF injections showed individual differences in the extent and density of labeling. This could be explained by the specific site and layer of the injection or frequency response of the cells at the site of the injection.

Injections in sites with frequencies of 5.6 $\mathrm{kHz}$ and $3.0 \mathrm{kHz}$ exhibited a denser labeling than injections in regions with frequencies of $1.0 \mathrm{kHz}, 1.5 \mathrm{kHz}$ and $2.5 \mathrm{kHz}$, regardless of the cortical representations for these frequencies. Low frequencies have more extended representations and they were supposed to exhibit a greater number of connections.

\section{Projections from $V$ and $D$ fields}

Projections from the $\mathrm{D}$ field to AAF were not clearly identified, neither were projections from the $\mathrm{V}$ field. In both cases, this might be explained by the fact that the site of the injections was very close to the edge of these small areas, diminishing the possibility of identifying two separate labeled areas. The V field could be even a part of AAF with a representation of low frequencies.

\section{Contralateral Projections}

After injections in AAF, projections to this area from the homotopic contralateral AAF (exhibiting the densest labeling), as well as from the heterotopic contralateral AI were observed. No contralateral projections were seen in the caudal fields or in the $\mathrm{V}$ or $\mathrm{D}$ fields. Homotopic and heterotopic projections showed a continuous labeling in a dorsoventral direction with an extension similar to the isofrequencies contours. Using injections of WGA-HRP in the $\mathrm{R}$ 
field of squirrels, contralateral projections were observed in the $\mathrm{R}$ field and peripheral regions (Luethke et al., 1988). In the owl monkey, contralateral projections from the rostral field were only seen in AI and R but not with the fields PL-CM and AM.

In the gerbil, a differentiation of callosal projections for homotopic fields (AAF) was confirmed, reaching particularly layer $\mathrm{V}$, while those from the heterotopic AI reached layers III to $\mathrm{V}$. In contrast to the owl monkey or the cat in which the anterior field has a separate labeling for its contralateral projections, in the gerbil no such pattern could be found, perhaps due to the small size of the auditory cortex.

\section{CONCLUSIONS}

The connection patterns seen after injections in AI and AAF, both ipsi- and contralateral are relatively constant with few deviations that could be explained by: i) differences in the preferred frequency in the injection sites that could have greater or lesser cortical representations; ii) injections in different cortical layers of the auditory field. A certain order can be observed in the cellular labeling patterns, which follow the isofrequency contours defined with $2 \mathrm{DG}$ in a dorsoventral direction. In the case of $\mathrm{AAF}$, an additional labeling orthogonal to the isofrequency was found. Additional differences in the projection patterns could derive from distinct sites of injection into the same isofrequency contour. Neurons with different localizations within an isofrequency contour might have different ways of projecting which represent more or fewer labeled cells depending on the case.

\section{ACKNOWLEDGMENTS}

This work was supported by grants FONDECYT 1961156 and DI-UACH S-96-12

\section{REFERENCES}

AITKIN L M, KUDO M, IRVINE DRF (1988) Connections of the primary auditory cortex in the common marmoset (Callithrix jacchus jacchus). J Comp Neurol 269:234-248

BENTIVOGLIO M, KUYPERS HGJM, CATSMANBRREVOETS CE, LOEWE H, DANN, O (1980) Two new fluorescent retrograde neuronal tracers which are transported over long distances. Neurosci Lett, 18:25-30

BRUGGE JF, IMIG TJ (1978) Some relationships of binaural response patterns of single neurons to cortical columns and interhemispheric connections of auditory area AI of cat cerebral cortex. In: NAUNTON, RF (ed) Evoked Electrical Activity in the Auditory Nervous System. New York: Academic Press. pp: 487-503

BRUGGE JF, REALE RA (1985) Auditory cortex. In: PETERS A, JONES EG (eds) Cerebral Cortex. Association and auditory cortices. New York: Plenum Press pp: 229-271

BUDINGER E, HEIL P, SCHEICH H (2000) Functional organization of auditory cortex in the Mongolian gerbil (Meriones unguiculatus). III. Anatomical subdivisions and corticocortical connections. Eur J Neurosci $12: 2425-2451$

CIPOLLONI PB, PANDYA DN (1989) Connectional analysis of the ipsilateral and contralateral afferent neurons of the superior temporal region in the rhesus monkey. J Comp Neurol 281:567-585

DIAMOND IT, JONES EG, POWELL TPS (1968) The association connections of the auditory cortex of the cat. Brain Res 11:560-579

EHRET G (1997) The auditory cortex. J Com. Physiol (A) 181:547-557

FITZPATRICK KA, IMIG TJ (1980) Auditory corticocortical connections in the owl monkey. J Comp. Neurol. 192: 589-610

IMIG TJ, RUGGERO MA, KITZES LM, JAVEL E, BRUGGE J F (1977) Organization of auditory cortex in the owl monkey (Aotus trivirgatus). J Comp Neurol 171:111-128

IMIG TJ, BRUGGE JF (1978) Sources and terminations of callosal axons related to binaural and frequency maps in primary auditory cortex of the cat. J Comp Neurol 182:637-660

IMIG TJ, REALE RA (1980) Pattern of cortico-cortical connections related to tonotopic maps in cat auditory cortex. J Comp Neurol 192: 293-332

IMIG TJ, REALE AR, BRUGGE F (1982) Patterns of cortical projections related to physiological maps in the cat. In: WOOLSEY CN (ed) Cortical Sensory Organization. Multiple Auditory Areas. Clifton: Humana Press. pp:1-57

JACOBSON S, TROJANOWSKI JQ (1974) The cells of origin of the corpus callosum in the rat, cat and rhesus monkey. Brain Res 74:149-155

JONES EG, COULTER JD, HENDRY SHC (1978) Intracortical connectivity of architectonic fields in the somatic sensory, motor and parietal cortex of monkey. J Comp Neurol 181:273-305

LUETHKE LE, KRUBITZER LA, KAAS JA (1988) Cortical connections of electrophysiologically and architectonically defined subdivisions of auditory cortex in squirrels. J Comp Neurol 268:181-203

LUETHKE LE, KRUBITZER LA, KASS JH (1989) Connections of primary auditory cortex in the new world monkey (Sanguinus). J Comp Neurol 285:487-513

MATSUBARA JA, PHILLIPS DP (1988) Intracortical connections and their physiological correlates in the primary auditory cortex (AI) of the cat. J Comp Neurol 268:38-48

MERZENICH MM, BRUGGE JF (1973) Representation of the cochlear partition on the superior temporal plane of the macaque monkey. Brain Res 50:275-296 
MONTERO VM (1980) Patterns of connections from the striate cortex to cortical visual areas in superior temporal sulcus of macaque and middle temporal gyrus of owl monkey. J Comp Neurol 189:45-59

MOREL A, GARRAGHTY PE, KASS JH (1993) Tonotopic organization, architectonic field and connections of auditory cortex in macaque monkeys. J Comp Neurol 335:437-459

RAUSCHECKER JP (1998) Parallel processing in the auditory cortex of primates. Audiol Neurootol 3:86-103

REALE RA, IMIG TJ (1980) Tonotopic organization in the auditory cortex of the cat. J Comp Neurol 192:265-291

REDIES H, SIEBEL U, CREUTFELDT OD (1989) Functional subdivisions in the auditory cortex of the guinea pig. J Comp Neurol 282:473-488

ROUILLER EM (1997) Functional organization of the auditory pathways. In: EHRET G, ROMAND R (eds) The central auditory system. New York: Oxford University Press. pp: 3-96
SCHEICH H, HEIL P, LANGER G (1993) Functional organization of auditory cortex in the Mongolian gerbil (Meriones unguiculatus). II. Tonotopic 2-deoxyglucose patterns. Eur J Neurosci 5:898-914

THOMAS H, TILLEIN J, HEIL P, SCHEICH H (1993) Functional organization of auditory cortex in the Mongolian gerbil (Meriones unguiculatus). I. Electrophysiological mapping of frequency representation and distinction of fields. Eur J Neurosci 5:882-897

WALLACE M, RUTKOWSKI R (2002) Interconnections of auditory areas in the guinea pig neocortex. Exp Brain Res 143:106-119

WINER JA (1984a) The pyramidal neurons in layer III of cat primary auditory cortex (AI). J Comp Neurol 229:476-496

WINER JA (1984b) Anatomy of layer IV in cat primary auditory cortex (AI). J Comp Neurol 224:535-567 